\title{
Sleep and epilepsy: a bidirectional influence
}

\begin{abstract}
Epilepsy and sleep are states that share neural mechanisms and processes that can affect each other. This tight pathophysiological relationship has very important consequences from the clinical point of view, which are well-known long time ago. However, the use of new techniques of study, from the neuronal level to the macroscopic system is increasing our knowledge of the real nature of the relation between sleep and epilepsy.

Keywords: epilepsy, communication network, emotion, synaptic plasticity, nocturnal seizures
\end{abstract}

Volume 2 Issue I - 2018

\author{
Jesús Pastor, Lorena Vega Zelaya \\ Clinical Neurophysiology, Hospital Universitario La Princesa, \\ Spain
}

Correspondence: Jesús Pastor, Neurofisiología Clínica, Hospital Universitario de La Princesa, C/Diego de León 62 , Madrid 28006, Spain, Tel 34-9I-52022I3, Fax 34-9I-40I3582, Email jesus.pastor@salud.madrid.org

Received: December 20, 2017 | Published: January 30, 2018
Abbreviations: CNS, central nervous system; NREM, nonrapid eye movement; REM, rapid eye movement; vEEG, video electroencephalography; PSG, polysomnography; IED, interictal epileptiform discharges; SSMA, supplementary sensorimotor area; JME, juvenile myoclonic epilepsy; TLE, temporal lobe epilepsies; NTLE, nocturnal temporal lobe epilepsy; SW, spike-wave ; PSW, polyspike-wave

\section{Introduction}

The central nervous system (CNS) can be considered as a communication network that controls every movement, thought, emotion, impression and memory. ${ }^{1}$ This process takes place normally, through the generation of continuously tiny electrical impulses in an orderly pattern. These impulses travel along the network of nerve cells, called neurons. A seizure occurs when the brain's neurons fire in an excessive and over synchronized manner, generating a sudden, uncontrolled surge of electrical activity in the brain. Epilepsy is the tendency toward recurrent, unprovoked seizures. ${ }^{2}$

It is an accepted fact that sleep consists of active brain states during which many biological processes occur, such as synaptic plasticity and memory consolidation. During non-rapid eye movement (NREM) sleep, the cells tend to discharge synchronously. Conversely, during rapid eye movement (REM) sleep and alert waking, cells discharge asynchronously. ${ }^{3}$

In the last two decades, the advance in video electroencephalography (vEEG) and polysomnography (PSG) techniques have raised awareness about the inherent relationship between sleep and epilepsy. ${ }^{4}$ So, we are now aware that epileptic activity disturbs sleep and sleep deprivation may aggravate epilepsy due to the decrease in seizure threshold thus forming a vicious circle. Besides, sleep clearly results in significant activation of both interictal epileptiform discharges (IEDs) and, for some definite kinds of epilepsies, of seizures too. In this necessarily short work, we briefly review some of the main facts and give our personal point of view about the relationships between both fields.

\section{Sleep induced epileptic activity and sleep disruption by epilepsy}

Sleep consists of repetitive cycles, advancing through NREM stages to REM. The seizure activating role of NREM sleep has been attributed to the thalamocortical circuitry responsible for the neuronal hyper synchronization of NREM sleep, which normally generates sleep spindles and high amplitude delta waves. It has been postulated that its effect is a significant increase of both interictal epileptiform discharges (IEDs) and seizures. ${ }^{4,5}$ In general, it is believed that IEDs are most prevalent during slow wave sleep, while seizures are more associated in lighter stages of NREM sleep. ${ }^{5}$

Research has shown a clear increase in firing rates and hyper synchronous burst-firing during both slow wave sleep and REM sleep in the epileptic focus, as compared to non-seizure generating regions. ${ }^{6,7}$ Probably, not only the thalamo-cortico-thalamic loop would be implicated, but changes in brain-stem neuromodulators can modify neuronal excitability and synchronicity.

During REM sleep and alert waking, cells discharge asynchronously thus; IEDs are relatively inhibited during REM sleep. In the same way, there is a lower probability of propagation of epileptic EEG discharges, probably because the synchronicity is a powerful condition to allow propagation..$^{8-10}$ In fact, it has been reported that IEDs observed during REM sleep are more restricted and usually more localizing to the epileptic focus. ${ }^{11}$

Sleep quality is usually affected in patients with epilepsy. Sleep schedule can be disturbed by their frequency of nocturnal seizures; the presence of coexisting sleep disorders and even by poor sleeps hygiene. Even generalized interictal epileptiform activity, in the absence of true seizures, can disturb the sleep pattern. Considerable sleep impairment in epileptic patients could negatively affect their quality of life and more important; impair their seizure control since sleep deprivation enhances cortical excitability. ${ }^{12}$

There is considerable evidence that severity of epileptiform activity during the sleep is associated to cognitive deficits. ${ }^{13}$ Therefore, 
it is extremely important to control this irritative activity, even in the absence of clinical seizures.

\section{Sleep related epilepsies}

Epilepsy should be considered more as a kind of pathologies, sharing several similarities, than a robust nosological entity. Therefore, not all the epilepsies are prone to present seizures during the sleep in the same degree. ${ }^{13}$

Frontal lobe seizures are more likely to arise from sleep than are temporal lobe seizures. Over one-third of partial seizures arise from sleep and they are usually related with medical intractability. Several epilepsy types are associated with sleep, some of them associate with significant cognitive impairment including, nocturnal frontal lobe epilepsy either supplementary sensorimotor area (SSMA) or autosomal dominant, benign epilepsy of childhood with centrotemporal spikes, juvenile myoclonic epilepsy (JME) and Lennox Gastaut syndrome. ${ }^{4}$ With respect to temporal lobe epilepsies (TLE), nocturnal temporal lobe epilepsy (NTLE) is a subtype of medically refractory focal epilepsy with nearly exclusive seizures at night-time and a good postoperative outcome.

There are some epilepsies, like JME, that tend to occur upon awakening. The interictal EEG shows diffuse or generalized spikewave (SW) and polyspike-wave (PSW) discharges, mostly seen at sleep onset and at awakening.

\section{Sleep disorders May Mimic or complicate epilepsy}

Excessive daytime sleepiness (EDS) is a very common complaint among epileptic patients. It is a frequent mistake attributes this symptom to antiepileptic drugs side effects. Many epilepsy therapies appear to have a negative effect on the microstructure of sleep, but also, epilepsy is associated with a reduction of REM sleep and stages 2 and 4 of sleep, leading to poorly restorative sleep and EDS. ${ }^{14}$

Identifying a sleep disorder associated with epilepsy is particularly important, since co-morbid primary sleep disorders may exacerbate seizure burden. Besides, it is not uncommon that sleep disorders like hypersomnia, insomnia, and parasomnias overlap and cause confusion with the differential diagnosis of epilepsy.

Finally, medical disorders such as nocturnal gastroesophageal reflux and nocturnal asthma or paroxysmal nocturnal dyspnoea from congestive heart failure also enter the differential diagnosis. Although, from the clinical point of view, usually it is easy to distinguish, we should not forget them.

\section{Conclusion}

Sleep and epilepsy have an interesting and clinically relevant relationship, with a bidirectional influence. Different stages of sleep modify the behaviour and frequency of EIDs and seizures. Thus, sleep may have a facilitating or precipitating effect on seizures. On the other hand, epilepsy itself could affect sleep pattern. What could result in poor control of seizures, because of sleep deprivation? Therefore, sleep may also have protective effect against epilepsy.

Finally, this strong relationship has a very important relevance from the clinical point of view, because it implies that the clinician at sleep unit must be well-trained in EEG recording and analysis, being familiar with epilepsy and bioelectrical manifestations of the different signatures of epilepsy during night recording, because there are several elements which are not epileptic but can be easily confounded, ${ }^{15}$ given place to a diagnostic mistake of extremely important consequences.

\section{Acknowledgements}

This work was financed by a grant from the Ministerio de Sanidad FIS PI17/02193 and was partially supported by FEDER (Fonds Europeen de Developpement Economique et Regional).

\section{Conflicts of interest}

The authors declared that there are no conflicts of interest.

\section{References}

1. Pastor J, Vega-Zelaya L. Redes neurales en epilepsia. Contribución iberoamericana a la epilepsia; 2008.

2. Fisher RS, Acevedo C, Arzimanoglou A, et al. ILAE official report: a practical clinical definition of epilepsy. Epilepsia. 2014;55(4):475-482.

3. Marks GA. The neurobiology of Sleep. Sleep A comprehensive handbook In: TL Lee-Chiong, editor. New Jersey, USA: John Wiley \& Sons; 2006. p. 11-18.

4. Al-Biltagi MA. Childhood epilepsy and sleep. World J Clin Pediatr. 2014;3(3):45-53.

5. St Louis EK. Sleep and epilepsy: strange bedfellows no more. Minerva Pneumol. 2011;50(3):159-176.

6. Staba RJ, Wilson CL, Fried I, et al. Single neuron burst firing in the human hippocampus during sleep. Hippocampus. 2002;12(6):724-734.

7. Staba RJ, Wilson CL, Bragin A, et al. Sleep states differentiate single neuron activity recorded from human epileptic hippocampus, entorihinal cortex, and subiculum. J Neurosci. 2002;22(13):5694-5704.

8. Pastor J, Navarrete EG, Sola RG, et al. Extrahippocampal desynchronization in non lesional temporal lobe epilepsy. Updates in temporal lobe epilepsy. Epilepsy Res Treatment. 2012;2012:1-9.

9. Palmigiano A, Pastor J, Sola RG. et al. Stability of synchronization clusters and seizurability in temporal lobe epilepsy. PLoS One. 2012;7(7):e41799.

10. Vega-Zelaya L, Pastor J, De Sola RG, et al. Disrupted ipsilateral network connectivity in temporal lobe epilepsy. PLoS One. 2015;10(10):e014085.

11. Sammaritano M, Gigli GL, Gotman J. Interictal spiking during wakefulness and sleep and the localization of foci in temporal lobe epilepsy. Neurology. 1991;41(2 Pt 1):290-297.

12. Del Felice A, Fiaschi A, Bongiovanni GL, et al. The sleep- deprived brain In normal and patients with juvenile myoclonic epilepsy: a perturbational approach to measuring cortical reactivity. Epilepsy Research. 2011;96:123131.

13. Shorvon SD. The clinical forms and causes of epilepsy. Handbook of Epilepsy treatment. Forms, causes and therapy in children and adults. 2nd ed. Massachussetts, USA: Blackwell; 2005. p. 1-59.

14. Bazil CW, Castro LH, Walczak TS. Reduction of rapid eye movement sleep by diurnal and nocturnal seizures in temporal lobe epilepsy. Arch Neurol. 2000;57(3):363-368.

15. Tatum WO, Husain AM, Benbadis SR, et al. Handbook of EEG interpretation. New York, USA: Demos Baltimor; 2008 